Original Research Article

\title{
Prescribing trends of fixed dose combinations in a tertiary care teaching hospital of Bhopal district
}

\author{
Rajanish Kumar Sankdia1, Mehul Agrawal ${ }^{2 *}$
}

${ }^{1}$ Department of Pharmacology, Bundelkhand Medical College, Sagar, Madhya Pradesh, India ${ }^{2}$ Department of Pharmacology, Gandhi Medical College, Bhopal, Madhya Pradesh, India

Received: 19 December 2018 Accepted: 29 January 2019

\section{*Correspondence to: \\ Dr. Mehul Agrawal, \\ Email: drmehulagrawal@ yahoo.co.in}

Copyright: (C) the author(s), publisher and licensee Medip Academy. This is an openaccess article distributed under the terms of the Creative Commons Attribution NonCommercial License, which permits unrestricted noncommercial use, distribution, and reproduction in any medium, provided the original work is properly cited.

\begin{abstract}
Background: Medicines are an integral part of healthcare. More than one drug is frequently used for treatment of either single ailment or multiple co-morbid conditions. Sometimes, two or more drugs are combined in a fixed ratio into a single dosage form, which is termed as fixed dose combinations (FDCs).

Methods: This prospective observational study was carried out in the patients admitted in various medical and surgical wards of Chirayu hospital associated with Chirayu medical college of Bhopal district for a period of 3 months. The prescribed FDCs were collected from the case sheet of the admitted patients. Patients of all ages and both sexes were included in the study.

Results: The total numbers of prescriptions from teaching hospital were 475 , out of which 373 patients $(78.52 \%)$ were prescribed FDCs. The first four most preferred FDCs were B complex preparations followed by pantoprazole + domperidone, Diclofenac+Paracetamol and Chlorpheniramine+Pseudoephedrine+Paracetamol.

Conclusions: In present study total 11types of FDCs were prescribed, out of 11 FDCs prescribed; only 1 type was enlisted in the essential drug list of WHO and Govt. of India. In only 4 types of FDCs there is scientific justification for combining ingredients. Most of FDCs do not have scientific justification for combining the ingredients. It is the need of the time that hospitals should constitute drugs and therapeutics review committees to promote rational prescription of FDCs.
\end{abstract}

Keywords: B complex preparations, Essential drug list, Fixed dose combinations, Prescription, Rationality, Teaching hospital

\section{INTRODUCTION}

Medicines are an integral part of healthcare. More than one drug is frequently used for treatment of either single ailment or multiple co-morbid conditions. Sometimes, two or more drugs are combined in a fixed ratio into a single dosage form, which is termed as fixed dose combinations (FDCs). The FDCs are justified when they demonstrate clear benefits in terms of (a) potentiating the therapeutic efficacy, (b) reducing the incidence of adverse effect of drugs, (c) having pharmacokinetic advantage, (d) better compliance by reducing the pill burden, (e) reducing dose of individual drugs, (f) decreasing development of resistance and $(\mathrm{g})$ cheaper than individual drug because of reduced cost from packaging to distribution. It is important that the above claims are adequately supported by scientific evidence. ${ }^{1}$ Rational drug use means patients receive medications appropriate to their clinical needs, in doses that meet their own individual requirements, for an adequate period of time and at the lowest cost to them and their community.

Some of the common irrational drug use problems are polypharmacy, overuse of injections, self medication, misuse of antibiotics, use of irrational fixed dose 
combinations (IFDCs) etc. $^{2}$ the rationality of FDCs should be based on certain aspects such $\mathrm{as}^{3}$ :

- The drugs in the combination should act by different mechanisms.

- The pharmacokinetics must not be widely different.

- The combination should not have supra-additive toxicity of the ingredients.

Most FDCs have the following demerits: ${ }^{3}$

- Dosage alteration of one drug is not possible without alteration of the other drug.

- Differing pharmacokinetics of constituent drugs pose the problem of frequency of administration of the formulation.

- By simple logic there are increased chances of adverse drug effects and drug interactions compared with both drugs given individually.

\section{METHODS}

This prospective observational study was carried out in the patients admitted in various medical and surgical wards of Chirayu hospital associated with Chirayu medical college of Bhopal district. Duration of study was approximately 3 months from January 2018 to March 2018. The prescribed FDCs were collected from the case sheet of the admitted patients. Patients of all ages and both sexes were included in the study.

Case sheet were prospectively screened and relevant data was entered in case record form. The prescription records was then divided into four categories for evaluation of prescribing pattern of FDCs in relation to age, sex, number of FDCs per prescription and prevalence order of most commonly prescribe FDCs.

The results were presented as either average or percentage. Available WHO essential medicine list 2015 and national essential medicine list 2015 were referred for evaluating the rationality of prescribed FDCs in the health care setting. Rationality and irrationality of FDCs was established on the basis of available scientific knowledge.

The study was undertaken only after ethical review and approval from ethics committee. The relevant information was collected from the patient case file and by no means was patient interviewed, the name of the patient was not included in the recording format to maintain the patient confidentiality and accordingly patient consent was not necessary. Data of all the patients was later analyzed by applying appropriate statistical measures.

\section{RESULTS}

The total numbers of prescriptions from teaching hospital were 475 , out of which 373 patients $(78.52 \%)$ were prescribed FDCs. On the basis of age, patients were categorized in age group of 0-15 year, 16- 30 year, 31-50 year and 51 year onwards. In this $9.53 \%$ patients were in $0-15$ year age group. In 16-30 year age group $18.2 \%$, in $31-50$ year age group $34.25 \%$, in 51 year onwards age group $38.04 \%$ patients were present.

In present study, $49.25 \%$ prescriptions contained one FDC, $38.16 \%$ contained two FDCs, $10.80 \%$ contained three FDCs, and $1.78 \%$ contained more than three FDCs.

Table 1: Prevalence order of commonly prescribed FDCs.

\begin{tabular}{|lll|}
\hline Name of combination & Number of prescriptions & Percentage of prescriptions \\
\hline B complex preparations & 324 & 68.2 \\
\hline Pantoprazole+domperidone & 248 & 52.2 \\
\hline Diclofenac+paracetamol & 136 & 28.6 \\
\hline Chlorpheniramine+pseudoephedrine+Paracetamol & 124 & 26.1 \\
\hline Amoxicillin+clavulanic acid & 119 & 25 \\
\hline Levocetrizine+monteleukast & 98 & 20.6 \\
\hline Oxethazaine +Aluminium hydroxide +Magnesium hydroxide & 54 & 11.36 \\
\hline Tramadol+paracetamol & 44 & 9.2 \\
\hline Calcitriol +Calcium citrate+Elemental zinc & 32 & 6.7 \\
\hline Aspirin+ Atorvastatin & 24 & 5 \\
\hline Ciprofloxacin+Dexamethasone & 12 & 2.5 \\
\hline
\end{tabular}

The first four most preferred FDCs were B complex preparations followed by pantoprazole \& domperidone, Diclofenac and paracetamol and Chlorpheniramine and pseudoephedrine and paracetamol (Table 1).

\section{DISCUSSION}

The total numbers of prescriptions were 475 , out of which 373 patients $(78.52 \%)$ were prescribed FDCs, this 
indicates that significant proportion of patients received FDCs.

In present study total 11types of FDCs were prescribed, Out of 11 types FDCs prescribed, only 1 type i.e. amoxicillin \& clavulanic acid was enlisted in the essential drug list of WHO and Govt. of India. In only 4 types of FDCs there is scientific justification for combining ingredients.

The first four most preferred FDCs were B complex preparations followed by pantoprazole \& domperidone, diclofenac \& paracetamol and chlorpheniramine \& pseudoephedrine \& paracetamol. Similar findings were published by Hindoliya et al and Rayasam et al in their research paper that the most commonly prescribed FDCs were B complex preparations. ${ }^{5.9}$

Possible explanation for the B complex prescribing can be that most of the patients reporting to the teaching hospital are from the low socioeconomic group and are suspected to have multiple vitamin deficiency, keeping this in mind it is a routing practice in the teaching hospital to prescribe vitamin B complex preparation to many patients. Moreover, the most commonly prescribed combination e.g. combinations of vitamin B complex series are ineffective, since all the molecules are absorbed by the same carrier which is saturable. ${ }^{4}$

Pantoprazole and domperidone combination was the second common prescribed FDC in our study. Similar findings were also reported in the study conducted by Rayasam et al. ${ }^{9}$ Proton Pump Inhibitors reduce gastric acid production in acid peptic diseases, which brings symptomatic relief. There is no justification of combining these drugs with antiemetic drug domperidone as peptic ulcer is not always associated with vomiting. Even in gastro-oesophageal reflux diseases (GERD), the domperidone is an inferior choice. GERD can be managed by not assuming the recumbence posture after $3 \mathrm{hrs}$ of food intake, elevating the head end, avoiding the acidic food stuffs and not taking the agents which relax the lower oesophageal sphincter. In drug therapy proton pump inhibitors represent excellent drugs followed by $\mathrm{H} 2$ blockers. Domperidone is even inferior to metoclopramide in GERD. There is no justification of combining inferior drug domperidone with excellent drugs like proton pump inhibitors or $\mathrm{H} 2$ blockers. ${ }^{5}$

Diclofenac and paracetamol is also an irrational drug combination because there is no justification of combining one NSAID with another NSAID having same pharmacological actions and mechanism of action. There is increased risk of nephrotoxicity when NSAIDs are combined. ${ }^{5}$

Chlorpheniramine and pseudoephedrine and paracetamol was given for cough and cold patients but again all the three drugs in this FDC are not required in every patient.
Leukotrine antagonist (montelukast) and levocetirizine combination was used in patients of asthma but leukotrine antagonist montelukast is used only as alternative to inhaled steroid in management of mild persistent asthma. There is no role of antihistaminic drug in asthma because it is not only histamine which is released from mast cell but there is release of various mediators from mast cell leading to bronchospasm and inflammation. ${ }^{5}$

Ciprofloxacin and dexamethasone eyedrops were given in some patients. This combination is also not justified as steroid should not be given in any patient unless specifically indicated. Opportunistic infections of the eye include bacterial, viral, and fungal infections and are most often associated with the use of topical ocular steroids. Glaucoma is more often associated with topical ocular or periocular steroids than with systemic steroids. ${ }^{6}$

Calcitriol, calcium citrate and elemental zinc FDC should not have been given without proper investigations and deficiency of all the ingredients.

This observation could be supported on following grounds:

1. In general, the medical representatives from pharmaceutical companies tend to influence the physicians prescribing behaviour by offering incentives in the form of cash or kind.

2. Continuous medical education (CME) programs which are conducted on regular basis in the teaching hospital update the knowledge of clinicians regarding the rational use of drugs and this has effect on prescribing behaviour in terms of rationality.

3. Most of the patients attending the teaching hospital belong to low socioeconomic background. Keeping affordability in mind they are usually prescribed from available hospital supply using background scientific information while selecting a drug. Moreover, these drugs are available free of cost to them. This keeps a check on unnecessary prescription and leaves little room for selecting and prescribing irrationally.

Most of the patients reporting to teaching hospital are from the low socioeconomic group and are suspected to have multiple vitamin deficiency. Therefore, it is a routine practice in the teaching hospital to prescribe vitamin B complex preparation to many patients.

India has a population of 120 crore plus. India has 4th largest pharmaceutical industry. In India there are 1 lakh formulations and around 1,000 basic drugs, including many irrational FDCs. In a study done by Gulati et al 12, over 70 dangerous FDCs are being sold in India under more than 1,000 brand names. Considering the enormous use of drugs in Indian population, it is the high time that pharmaceutical companies, health care professionals and 
regulatory authorities join hands and prescribe guidelines for the manufacture and sale of FDCs. ${ }^{7}$

India is the country with significant drug use problems. There is a concern regarding the irrational production, prescription, and use of FDCs. The rationality of a fixed dose combination is the most controversial and debated issue in today's clinical practice. The Indian laws have not been properly defined to grant marketing approvals for the FDCs by state or central drug controlling authorities. Therefore, the state drug controlling authorities have continuously been approving various FDCs, lacking any pharmacodynamic or pharmacokinetic advantages and acceptable rationale. ${ }^{8}$

The FDCs formulated without due diligence can pose problems namely (a) pharmacodynamic mismatch between the two components, one drug having additive/antagonistic effect leading to reduced efficacy or enhanced toxicity, (b) pharmacokinetic mismatch and having peak efficacy at different time, (c) chemical no compatibility leading to decreased shelf life, (d) drug interactions because of the common metabolizing pathways, and (e) limitations of finer dosing titration of individual ingredients.

Although FDCs are available in almost all therapeutic categories, many of them are bizarre combinations. The therapeutic categories having high number of FDCs are cough, cold, and fever preparations; analgesics and muscle relaxants; antimicrobials; drugs for hypertension, dyslipidemia, diabetes, and psychiatric disorders; and vitamins and minerals. The FDC formulation may have up to 5 or even more ingredients with or without rationality of their presence and in the quantity. ${ }^{1}$

FDCs should be developed to increase efficacy, reduce toxicity and for better patient compliance. The usage of these irrational FDCs can lead to increased adverse drug reactions, unnecessary hospitalization and financial burden. While for the prescriber it is difficult to individualize the dose and he/she could face problems when subjected to litigation in the consumer court, as these combinations do not find reference in standard textbooks or acclaimed journals. ${ }^{9}$

The reason behind the use of FDCs is that they improve clinical outcomes, reduce resource use, and cost effectiveness. $^{10}$

All the health care providing institutions especially teaching hospitals should focus on the teaching of FDCs to all the budding doctors starting from inception i.e. graduation level training with the following suggestions: 1.There should be due emphasis on FDCs during teaching Pharmacology. 2. All clinical departments should adopt rational practice of prescribing FDCs 3.Emphasis on developing rational and ethical thinking among undergraduates during training about clinical practice to prescribe FDCs. ${ }^{11}$
Irrational FDCs are prescribed by all the departments. Physicians were ignorant about the essential drugs and FDCs. Patients didn't have access to rational medicines. Therefore, physicians and regulators should be alerted in time. Regulatory actions or government laws should be made mandatory. Availability and access to 348 essential medicines for basic health care should be the priority of the government. Implementation of central drug standard control organisations (CDSCO) guidelines on industries for manufacture of FDCs must be made compulsory. ${ }^{12}$

\section{CONCLUSION}

In present study total 11types of FDCs were prescribed, Out of 11 FDCs prescribed, only 1 type was enlisted in the essential drug list of WHO and Govt. of India. In only 4 types of FDCs there is scientific justification for combining ingredients. Most of FDCs do not have scientific justification for combining the ingredients. It is the need of the time that hospitals should constitute drugs and therapeutics review committees to promote rational prescription of FDCs.

Funding: No funding sources

Conflict of interest: None declared

Ethical approval: The study was approved by the Institutional Ethics Committee

\section{REFERENCES}

1. Gupta YK, Ramachandran SS. Fixed dose drug combinations: Issues and challenges in India. Indian J Pharmacol. 2016;48:347-9.

2. Kadir Alam, Arjun Poudel, Subish Palaian. Fixed Dose Combination antimicrobials practices in Nepal. J Clin Diagnos Res. 2010;4:3255-60.

3. Amitava S. Indian market's fixation with fixed dose combinations (Editorial). Rational Drug Bulletin. 2002;12: 1 .

4. Anand S, Asha A.N, Uday Bhosale, Sarasija Suresh. Emergence of irrationality in fixed dose combinations. Pharma Times. 2008;40:17-21.

5. Dhaneria SP. Rational and irrational drug combinations. Drug bulletin. Department of pharmacology Post Graduate Institute Med Educ Res Chandigarh (India). 2010;35:2.

6. Renfro L, Snow JS. Ocular effects of topical and systemic steroids. Dermatol Clin. 1992;10(3):505-12.

7. Hindoliya M, Sharma PK, Dhaneria SP. Prescribing Trends of Fixed Dose Combinations in Teaching and Non Teaching Hospitals of Ujjain District. J Pharm Res. 2012,5(7),3503-5.

8. Jaina NK, Akarte AB, Deshmukhb PT, Kannojiac P, Garudc N, Akasha Y. Rationality Of Fixed Dose Combinations: An Indian Scenario. The Pharma Res. 2009;01:158-68.

9. The Use Of Essential Drugs. WHO Technical Report Series 825. Geneva. World Health Organization. Available at apps.who.int/medicinedocs/ 
documents/s16063e/s16063e.pdf. Accessed on 22 June 2018.

10. Angus DC, Walter T, Linde-Zwirble S, Tam W, Ghali JK, Sabolinski ML, et al. Winkelmayer, Manuel Worcel. Cost-effectiveness of fixed dose combination of isosorbide dinitrate and hydralazine therapy for blacks with heart failure. Circulation. 2005; 112:3745-53.

11. Upadhyay D, Singh A, Joshi HS, Agarwal M, Katyal R. Study of Prescription Patterns of Fixed Dose Combinations Prescribed by Medical Interns. Int $\mathbf{J}$ Biomed Res. 2016;7(9):624-8.
12. Rayasam SP, Dudhgaonkar SS, Dakhale GN, Hire RC, Deshmukh PS, Gaikwad NN. The irrational fixed dose combinations in the Indian drug market: an evaluation of prescribing pattern using WHO guidelines. Int J Basic Clin Pharmacol. 2013;2(4):1.

Cite this article as: Sankdia RK, Agrawal M.

Prescribing trends of fixed dose combinations in a tertiary care teaching hospital of Bhopal district. Int $\mathrm{J}$ Basic Clin Pharmacol 2019;8:507-11. 